\title{
АВТОМАТИЗИРОВАННОЕ УПРАВЛЕНИЕ ПРОЦЕССОМ БОРНОГО РЕГУЛИРОВАНИЯ В ЯДЕРНЫХ РЕАКТОРАХ ТИПА ВВЭР-1000
}

\author{
Цисельская Т.А. ${ }^{1}$, Ковтун А.В. ${ }^{1}$ \\ ${ }^{1}$ Одесский национальный политехнический университет, г.Одесса
}

Copyright (C) 2014 by author and the journal “Automation technological and business - processes”. This work is licensed under the Creative Commons Attribution International License (CC BY). http://creativecommons.org/licenses/by/4.0/

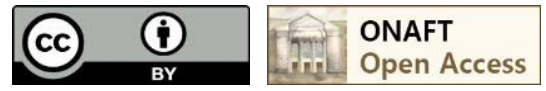

DOI: $10.15673 / 2312-3125$.

\begin{abstract}
Аннотация
Рассмотрена актуальная задача создания системы автоматизированного управления процессом борного регулирования в реакторах типа ВВЭР-1000. Результаты решения этой задачи были использованы для дальнейшей разработки компьютерно-интегрированной системы управления, которая позволяет увеличить эффективность решений, принимаемых оперативным персоналом в процессе управления реакторной установкой.
\end{abstract}

\section{Abstract}

An actual task of creating automated control system of boron regulation in WWER-1000 is considered. The results to this problem were used for the further development of computer-integrated control system that makes the decisions, taken by operational staff in the reactor control, be more efficient.

Ключевые слова

Борное регулирование в реакторах типа ВВЭР-1000, система управления, математическая модель, оптимизация процесса, прогноз параметров.

Введение. Основной целью эксплуатации АЭС в Украине является производство электроэнергии. Наилучшим режимом работы ядерных реакторов с точки зрения безопасности и надежности является режим работы на номинальном уровне мощности. Но на практике энергосистема накладывает свои требования и, следовательно, необходимо обеспечение возможности менять мощность энергоблока, чтобы обеспечить режим следования за нагрузкой. Поэтому к современным системам автоматизации реакторных установок предъявляются повышенные требования, что в первую очередь касается алгоритмов управления технологическими процессами, которые должны обеспечивать надежные и безопасные условия эксплуатации.

Приоритетными остаются вопросы управления реакторными установками с водо-водяными энергетическими реакторами (ВВЭР) единичной мощностью 1000 МВт, в которых непосредственно происходит ядерная реакция: в случае аварии реакторные установки являются основными источниками радиоактивного загрязнения окружающей среды.

Применение борного регулирования для управления реакторами типа ВВЭР-1000 позволяет увеличить время работы на максимальной мощности, обеспечивая экономическую эффективность эксплуатации энергоблока, а также поддерживать РУ в нужных безопасных пределах, которые делают невозможным возникновение и развитие аварийных ситуаций [1].

Доступные в литературных источниках методы управления процессом борного регулирования на АЭС Украины имеют недостатки, обусловленные недостаточной информационной поддержкой оперативного 


\section{1 ПИТАННЯ ТЕОРІї, МЕТОДИ ТА АЛГОРИТМИ ЕФЕКТИВНОГО АВТОМАТИЧНОГО УПРАВЛІННЯ ОБ’ЄКТАМИ ХІМІКО-ТЕХНОЛОГІЧНОГО ТИПУ}

персонала. К перечисленным недостаткам можно отнести отсутствие эффективных критериев качества процесса, методики оценки текущей ситуации. У оператора, который осуществляет борное регулирования, отсутствуют четкие инструкции, регламентирующие проведение процесса, а также возможность оценки и прогноза предполагаемых действий [2].

Как в зарубежных, так и в украинских работах последних 5-ти лет просматривается тенденция развития основных направлений оптимизации управления мощностью и энергораспределением путем увеличения роли борного регулирования в режимах нормальной эксплуатации.

Необходимо отметить, что возникновение аварийных ситуаций на АЭС может привести к тяжелым экономическим и экологическим последствиям. Поэтому при разработке и реализации алгоритмов управления процессом борного регулирования целесообразно прогнозировать изменение основных параметров работы энергоблока с использованием систем имитационного моделирования.

Чрезвычайно важным обстоятельством является тот факт, что атомная энергетика доказала свою экономическую эффективность практически во всех районах земного шара. Кроме того, даже при большом масштабе энергопроизводства на АЭС атомная энергетика не создаст транспортных проблем, поскольку требует малых транспортных расходов, освобождает общество от постоянных перевозок органического топлива. В настоящее время на АЭС Украины эксплуатируется одиннадцать РУ с ВВЭР-1000. Они входят в состав Запорожской, Ровенской, Хмельницкой и Южно-Украинской АЭС. Последние генерируют около 93\% электроэнергии украинских АЭС и позволяют сэкономить более 28 тонн угля или 16 млн. тонн нефти ежегодно [3].

Изложение основного материала. На рис.1 изображена принципиальная схема 2-контурной АЭС с реактором 1, парогенератором (ПГ) 2, турбиной 3 и циркуляционными насосами 5 и 6 с обозначением физических величин: $t_{1 T}$ - температура теплоносителя на входе в реактор; $t_{2 T}$ - температура теплоносителя на выходе из реактора; $t_{\Pi \Gamma}^{B x}-$ температура теплоносителя на входе в ПГ; $t_{\Pi \Gamma}^{B b x}-$ температура теплоносителя на выходе из ПГ; $D-$ расход пара; $t_{S}-$ температура насыщенного пара; $i_{\Pi B}-$ энтальпия питательной воды [4].

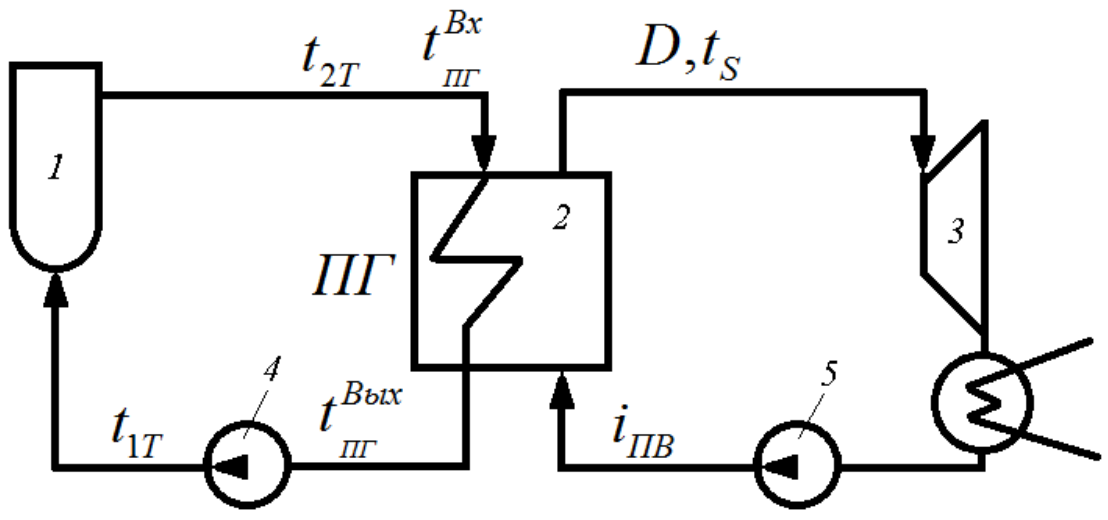

Рис. 1. Технологическая схема 2-контурной АЭС

Ядерный реактор описывается уравнениями кинетики, связывающим реактивность $\rho$ с относительной плотностью нейтронов $n$, и уравнением температурного эффекта реактивности по теплоносителю и топливу. Кроме того, в ядерных энергетических установках (ЯЭУ) включены следующие структурные элементы (рис.2) [5]: ядерное топливо (горючее) - 2; оболочка тепловыделяющего элемента - 3; теплоноситель в реакторе - 4; трубопроводы к реактору и ПГ -5 и обратно - 6; ПГ по теплоносителю первого контура -7 ; ПГ по теплоносителю второго контура - 8 . Физические величины: $t_{f}$ - температура топлива; $t_{o б}$ - температура оболочки; $\Delta \rho-$ изменение реактивности; $\rho_{6 H}-$ внешняя реактивность. 


\section{$\underline{1}$ ПИТАННЯ ТЕОРІї, МЕТОДИ ТА АЛГОРИТМИ ЕФЕКТИВНОГО АВТОМАТИЧНОГО УПРАВЛІННЯ ОБ’ЄКТАМИ ХІМІКО-ТЕХНОЛОГІЧНОГО ТИПУ}

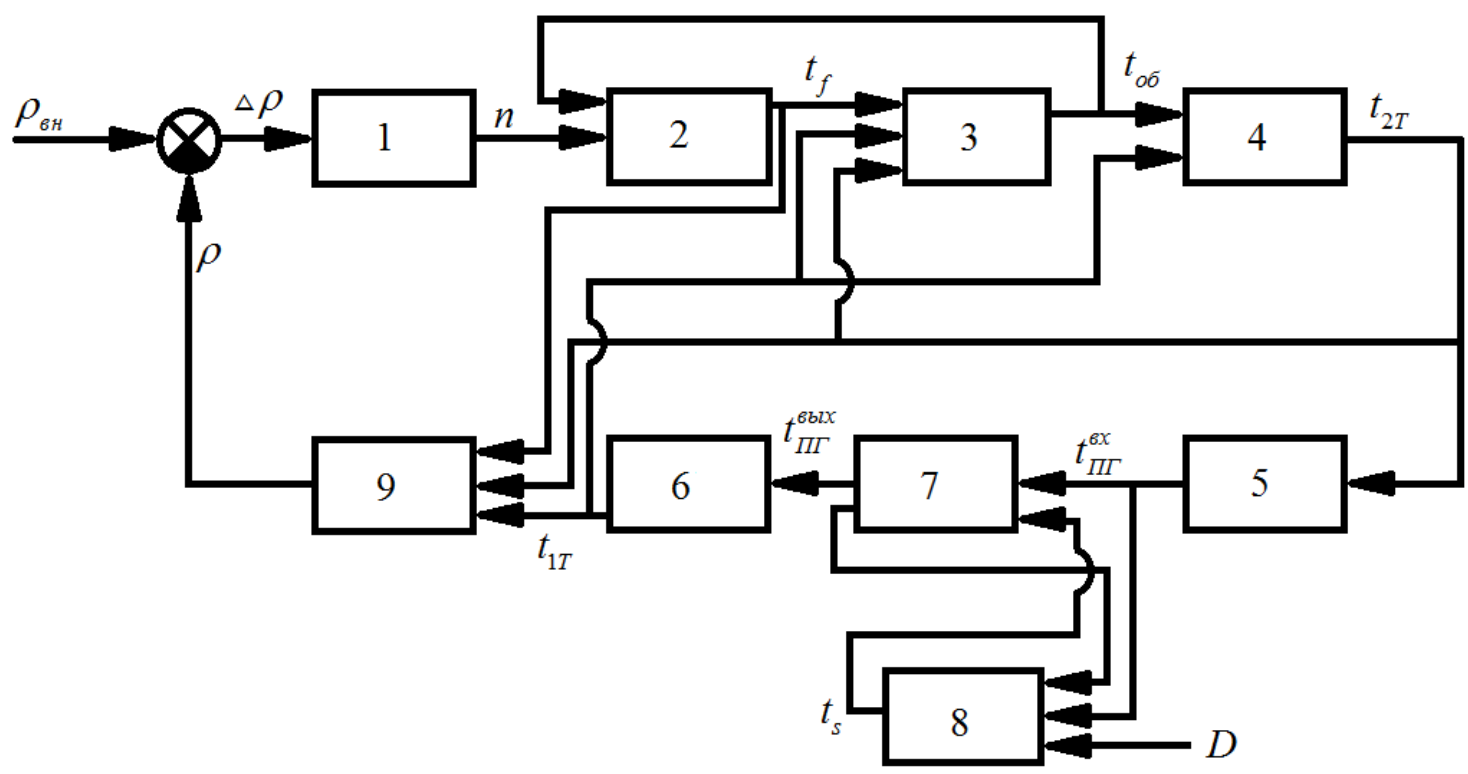

Рис. 2. Структурная схема энергоблока

На практике борное регулирование (БР) заключается в дискретном вводе определенного количества концентрированной борной кислоты КБК или чистого конденсата (ЧК) до получения желаемого результата. Оценку своих действий по БР оперативный персонал осуществляет, как правило, по изменению уровня мощности, аксиального офсета и значениям коэффициентов неравномерности энерговыделения, а не по значению концентрации борной кислоты [5].

График изменения концентрации БК при существующем на практике способе проведения БР имеет ступенчатый вид (рис. 3, кривая 1). Результаты исследований ведущих ученых РНЦ «Курчатовский институт» говорят о том, что изменение концентрации должно быть монотонным (см. рис.3, кривая 2).

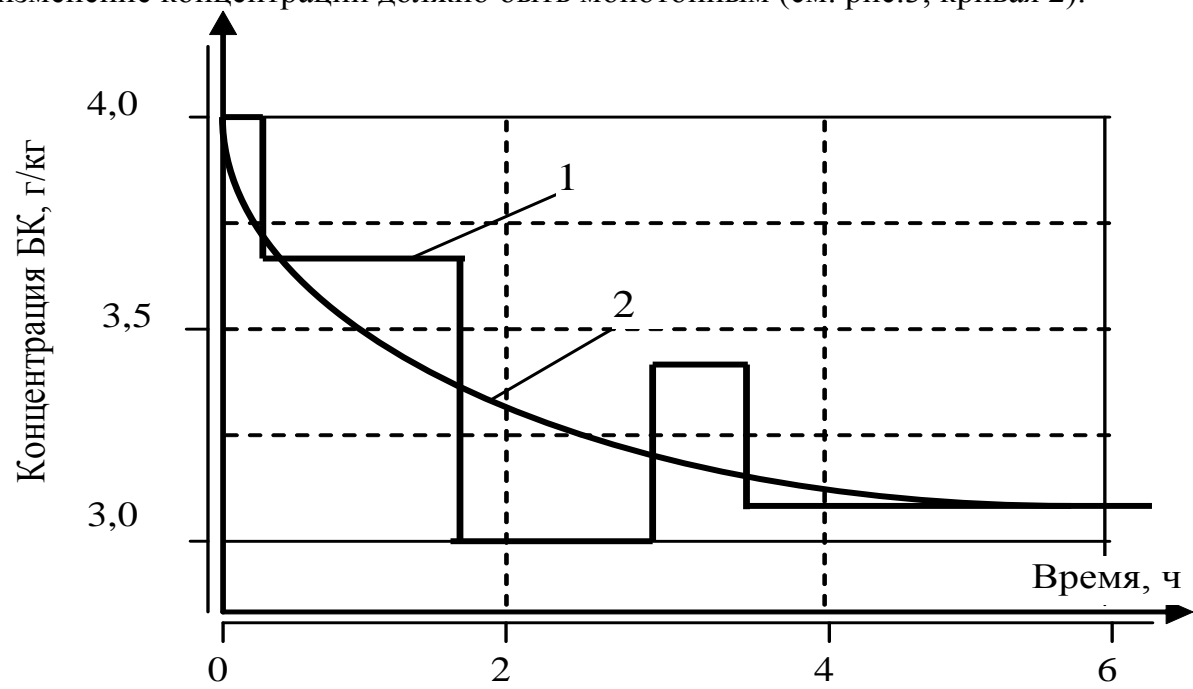

Рис. 3. Изменение концентрации борной кислоты в теплоносителе первого контура: 1 - ступенчатое изменение концентрации БК; 2- монотонное.

В случае монотонного изменения концентрации борной кислоты количество жидких радиоактивных отходов снижается (рис.4). 


\section{1 ПИТАННЯ ТЕОРІЇ, МЕТОДИ ТА АЛГОРИТМИ ЕФЕКТИВНОГО АВТОМАТИЧНОГО} УПРАВЛІННЯ ОБ’ЄКТАМИ ХІМІКО-ТЕХНОЛОГІЧНОГО ТИПУ

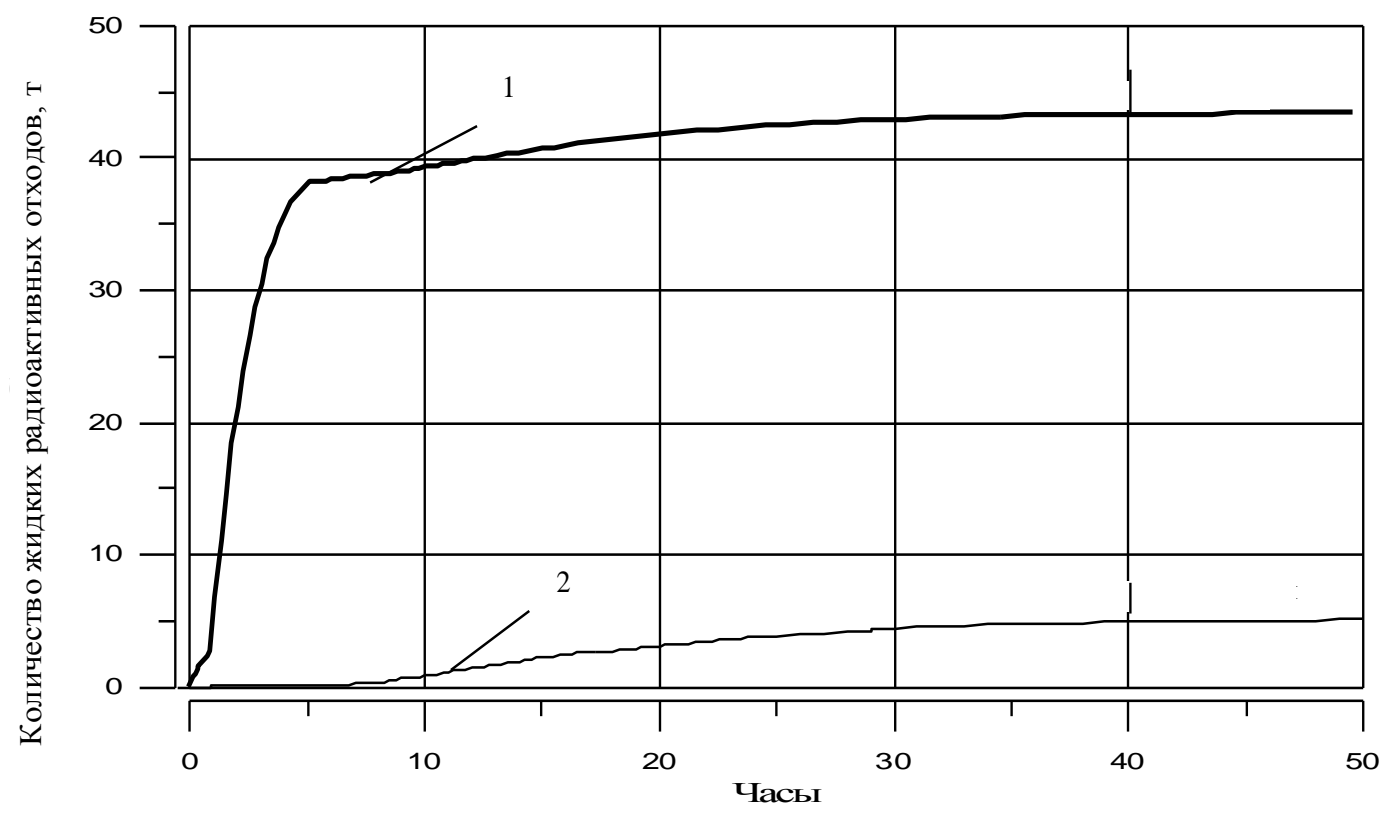

Рис. 4. График накопления жидких радиоактивных отходов: 1- ступенчатое изменение концентрации БК в первом контуре; 2 - монотонное.

Таблица 1

Условия стационарного состояния активной зоны (АЗ) реактора

a) тепловая мощность реактора поддерживается автоматическим регулятором мощности (АРМ) с точностью $\pm 2 \%$ от номинальной $N_{\text {ном }}$;

б) рабочая группа ОР СУЗ поддерживается в пределах регулировочного диапазона перемещений (70 90\% от низа АЗ);

в) величины измеренных с помощью штатной системы внутриреакторного контроля (СВРК) коэффициентов неравномерности $K v$ не превышают значений $K_{v}(i, j) \leq K_{v}^{n p e d}(i, j)$, где $\mathrm{i}-$ номер точки измерения по высоте АЗ реактора $(i=1,2,3, \ldots .7) ; \mathrm{j}$ - ТВС А3 реактора $(j=1,2,3 \ldots . .163)$

г) величина измеренного с помощью штатной СВРК АО находится в диапазоне: $-0,02 \leq A O \leq 0,15$

Признаком нарушения стационарного состояния является изменение реактивности, что, согласно требованиям технологического регламента безопасности (ТРБ), является сигналом к началу проведения борного регулирования. Поэтому изменение реактивности предложено использовать в качестве критерия оптимального управления процессом борного регулирования [6].

Текущее изменение реактивности реактора при работе на номинальном уровне мощности представляет собой такую алгебраическую сумму:

$$
\Delta \rho=\Delta \rho_{N}+\Delta \rho_{T^{\circ}}+\Delta \rho_{\text {ОРСУЗ }}+\Delta \rho_{б о р},
$$

где $\Delta \rho_{N}$ - вклад реактивности, обусловленный изменением уровня мощности от $\mathrm{N}_{1}$ до $\mathrm{N}_{2}, \%$;

$\Delta \rho_{T^{\circ}}$ - вклад реактивности, вызванный изменением средней температуры теплоносителя в реакторе от $\mathrm{T}_{1}{ }^{\circ} \mathrm{C}$ до $\mathrm{T}_{2}{ }^{\circ} \mathrm{C}, \%$;

$\Delta \rho_{О Р С \text { З }}$ - вклад реактивности, который определяется изменением положения рабочей группы ОР СУЗ от $\mathrm{H}_{1}$ до $\mathrm{H}_{2}, \%$;

$\Delta \rho_{\text {бор }}$ - вклад реактивности, зависящий от изменения концентрации борной кислоты в контуре от $C_{1}^{(1)}$ до $C_{1}^{(2)}$,\%; 


\section{I ПИТАННЯ ТЕОРІЇ, МЕТОДИ ТА АЛГОРИТМИ ЕФЕКТИВНОГО АВТОМАТИЧНОГО УПРАВЛІННЯ ОБ’ЄКТАМИ ХІМІКО-ТЕХНОЛОГІЧНОГО ТИПУ}

Составляющие (2) для любого момента времени известны и могут приобретать как положительные, так и отрицательные значения в зависимости от начального и конечного уровня мощности, средней температуры теплоносителя, положение ОР СУЗ и концентрации борной кислоты в теплоносителе. Суммарное изменение реактивности реактора $\Delta \rho$ также может приобретать как положительные, так и отрицательные значений. Оперативный персонал должен поддерживать реактор в критическом состоянии, при котором $\Delta \rho=0$. Нарушение условий, приведенных в табл. 1, вызывает изменения реактивности реактора и в общем случае определяется суммой первых трех слагаемых выражения (1):

$$
R=\Delta \rho_{N}+\Delta \rho_{T^{\circ}}+\Delta \rho_{O P C У 3},
$$

i $R \neq 0$.

Возвращение реактора в критическое состояние осуществляется путем введения противоположного по знаку изменения реактивности за счет увеличения или уменьшения концентрации борной кислоты в теплоносителе первого контура. Учитывая изложенное, целевую функцию можно изобразить так:

$$
I=\int_{0}^{t}\left(R-\Delta \rho_{б о р}\right)^{2} d t \rightarrow \min ,
$$

Минимизируемый функционал процесса борного регулирования получен в следующей форме [7]:

$$
\begin{aligned}
I & =\alpha_{\text {бор }}^{2} \frac{\left[C_{1}(0)-C_{2}\right]^{2}}{2 A} \cdot \exp (2 A t)+ \\
& +2 \alpha_{\text {бор }} \frac{C_{1}(0)-C_{2}}{A} \cdot\left[\alpha_{\text {бop }}\left(C_{2}-C_{1}(0)\right)-R\right] \cdot \exp (A t)+ \\
& +\left\{\left(R-\alpha_{\text {бop }} C_{2}\right)^{2}-\alpha_{\text {боp }} C_{1}(0) \cdot\left[2 R-\alpha_{\text {бор }} C_{1}(0) C_{2}+2\right]\right\} \cdot t- \\
& -\alpha_{\text {бор }} \frac{C_{1}(0)-C_{2}}{A}\left\{\frac{3 \alpha_{\text {боp }}\left[C_{1}(0)-C_{2}\right]}{2}-2 R\right\} \rightarrow \min
\end{aligned}
$$

где $\alpha_{\text {бор }}^{2}$ - дифференциальная эффективность борной кислоты, \% / $/$ кг;

$C_{1}(0)$ - концентрация борной кислоты в 1-м контуре в начальный момент времени, г / к2;

$C_{2}$ - концентрация борной кислоты в подпиточной воде, г / кг;

$A=G_{1} / V_{1} ; \quad G_{1}$ - расход подпитки, м $^{3} / 4 ;$

$V_{1}$ - объем теплоносителя в 1 -м контуре, $\mu^{3}$;

$R$ - изменение реактивности, вызванное нарушением стационарного состояния АЗ, \%.

Граничные условия:

$$
\begin{aligned}
0 \leq C_{2} & \leq 162 / \kappa 2, \\
t & >0,
\end{aligned}
$$

Поскольку $I=f\left[t\left(C_{2}\right)\right]$, то необходимым условием экстремума функционала $I$ является $\frac{d I(t)}{d t}=0$ :

$$
\begin{gathered}
y^{2}+2\left[\frac{C_{2}-C_{1}(0)}{C_{1}(0)-C_{2}}-\frac{R}{\alpha_{\text {бop }}\left(C_{1}(0)-C_{2}\right)}\right] \cdot y+\frac{R^{2}}{\alpha_{\text {бop }}^{2}\left(C_{1}(0)-C_{2}\right)^{2}}- \\
-\frac{2 R\left(C_{1}(0)+C_{2}\right)}{\alpha_{\text {бop }}\left(C_{1}(0)-C_{2}\right)^{2}}+\frac{\left(C_{2}-C_{1}(0)\right)^{2}}{\left(C_{1}(0)-C_{2}\right)^{2}}=0
\end{gathered}
$$

где $y=\exp (A t)$. 


\section{1 ПИТАННЯ ТЕОРІЇ, МЕТОДИ ТА АЛГОРИТМИ ЕФЕКТИВНОГО АВТОМАТИЧНОГО} УПРАВЛІННЯ ОБ'ЄКТАМИ ХІМІКО-ТЕХНОЛОГІЧНОГО ТИПУ

В результате анализа корней уравнения (6) получено выражение для нахождения оптимального времени процесса борного регулирования:

$$
t=-\frac{V_{1}}{G_{1}} \ln \left(\frac{R-\alpha_{\text {бор }}\left(C_{2}-C_{1}(0)\right)-4 \sqrt{R \alpha_{\text {бор }} C_{1}(0)}}{\alpha_{\text {бор }}\left(C_{1}(0)-C_{2}\right)}\right),
$$

Задача оптимального управления процессом борного регулирования свелась к отысканию такого значения концентрации $C_{2}$ борной кислоты в подпиточной воде, когда функция (8) достигла бы положительного минимального значения $(t>0)$ и обеспечила бы минимум функционала (4) с использованием необходимых и достаточных условий экстремума функции одной переменной [8].

Учитывая изложенное выше, алгоритм синтеза оптимального управления процессом борного регулирования в процессе функционирования РУ в реальном времени с учетом изменения реактивности должен включать следующие основные операции:

1. Оценка текущего состояния активной зоны реактора в дискретные моменты времени, соответствующие началу очередного цикла формирования управления, путем расчета изменения реактивности по формуле (1).;

2. В случае выхода реактора из критического состояния необходимо найти минимум функции (5) с учетом ограничений (5) и (6). Результатом решения является значение времени переходного процесса $t$ и концентрации борной кислоты $C_{2}$ в подпиточной воде.;

3. Вычисление конечного значения концентрации $C_{l}(t)$ в теплоносителе первого контура методом РунгеКутта на интервале $[0, t]$ с использованием найденных значений $t$ и $C_{2}$. Если $C_{l}(t)<C_{l}(0)$, то подпитку необходимо производить чистым конденсатом, иначе - раствором концентрированной борной кислоты.

4. Расчет объема чистого конденсата или концентрированной борной кислоты, который необходимо ввести для возврата реактора в критическое состояние, по формуле:

где $V_{\text {подп }}$ - объем подпитки, $\mu^{3}$;

$$
V_{\text {nодn }}=G_{1} \cdot t
$$

$G_{l}$ - расход подпитки в теплоноситель первого контура, $M^{3} / 4$;

$t$ - время, в течение которого необходимо осуществлять подпитку, 4 .

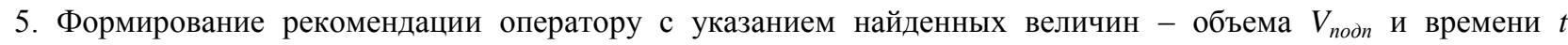
подпитки, концентрации борной кислоты $C_{2}$ в подпиточной воде, конечного значения концентрации БК $C_{l}(t)$ в теплоносителе первого контура.

Результаты. Любое отклонение одного или нескольких параметров состояния АЗ от значений, приведенных в таблице 1 , в работе принято называть событием.

Изменение параметров АЗ исследовались в режиме поддержания постоянного уровня мощности. Экспериментальные расчеты осуществлялись с помощью системы управления процессом борного регулирования и проходили в два этапа. На первом этапе прогноз осуществлялся с учетом рекомендации, генерируемой системой управления, на втором - без учета рекомендации. Полученные в процессе экспериментальных исследований результаты сравнивались с реальными данными. Индексом «1» отмечены результаты, полученные на первом этапе, индексом «2» - на втором этапе, «ИСХ» - архивные данные.

Исходное событие: в процессе работы реактора произошло снижение уровня мощности с 2941 МВт (98,03\%) до 2919 МВт (97,3\%) вследствие выгорания топлива, то есть отклонение от номинального уровня мощности составило более $2 \%$. Автоматический регулятор мощности (АРМ) извлек рабочую группу ОР СУЗ, что вызвало увеличение уровня мощности до 2955 МВт (98,5\%). Согласно требованиям ТРБ, оператор должен периодически возвращать группу в исходное состояние с помощью системы БР. 


\section{$\underline{1}$ ПИТАННЯ ТЕОРІЇ, МЕТОДИ ТА АЛГОРИТМИ ЕФЕКТИВНОГО АВТОМАТИЧНОГО} УПРАВЛІННЯ ОБ’ЄКТАМИ ХІМІКО-ТЕХНОЛОГІЧНОГО ТИПУ

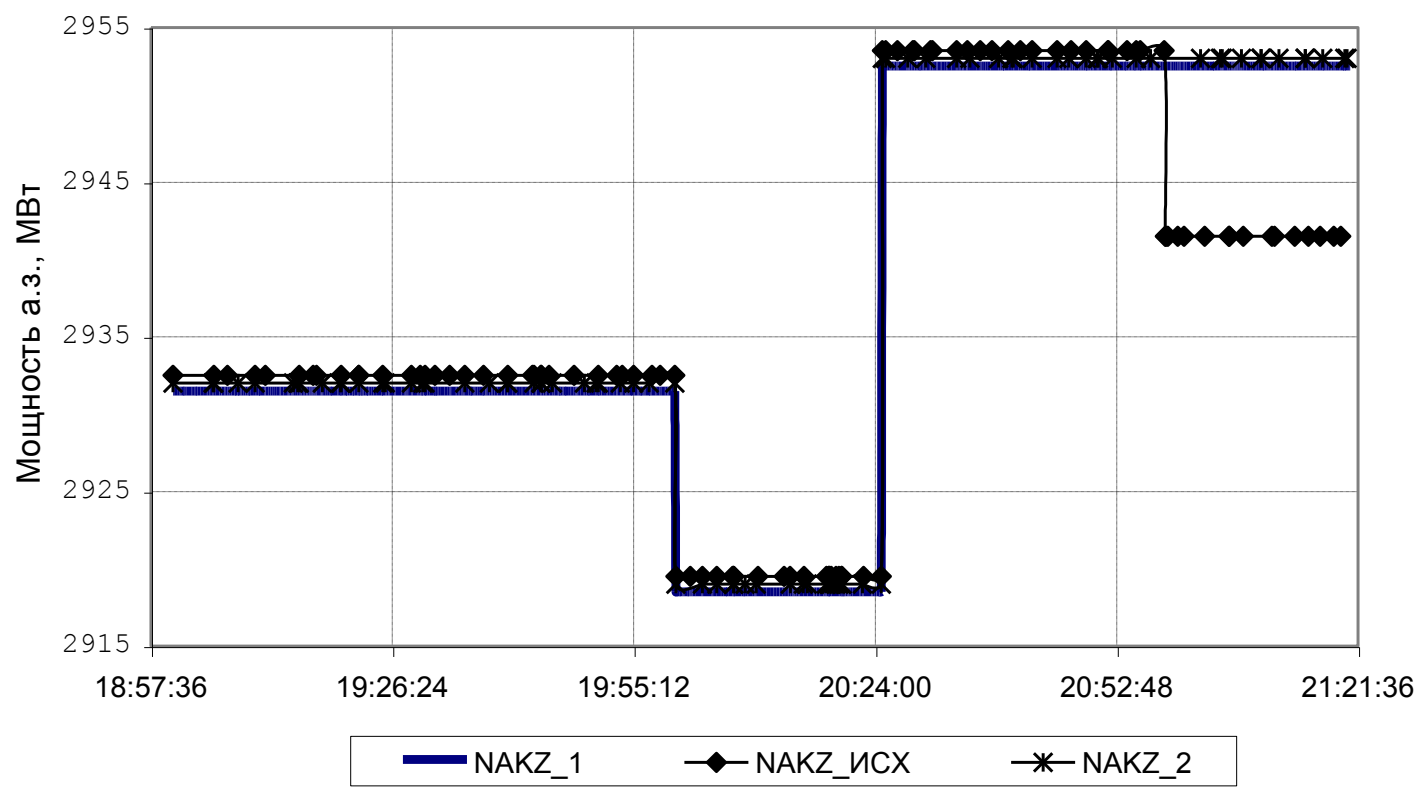

Рис. 5. Изменение уровня мощности в процессе БР

После срабатывания АРМ значение уровня мощности (рис. 5, 20:18) вернулось в диапазон, указанный в табл. 1. Согласно требованиям регламента, оператор должен вернуть группу в исходное положение, изменив концентрацию борной кислоты. Однако оператор снизил концентрацию в первом контуре (рис. 7, 20:58) без изменения положения рабочей группы ОР СУЗ. Изменение реактивности, как показано на рис. 8, в этом случае не минимизируется.

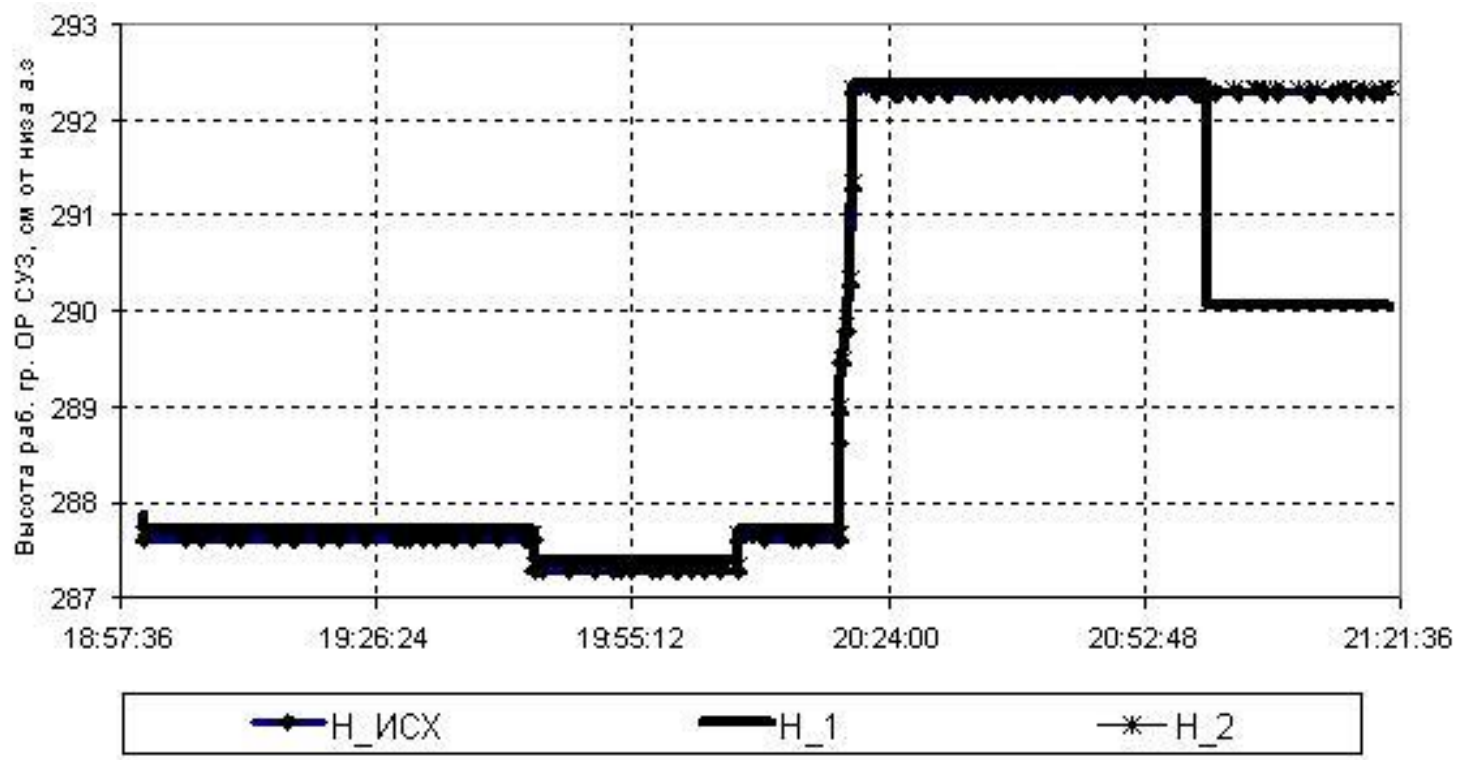

Рис. 6. Положение рабочей группы ОР СУЗ в процессе БР

Решение, предложенное системой управления, предполагает возврат группы в исходное положение путем снижения концентрации борной кислоты в первом контуре (21:00). После выполнения предложенных действий изменение реактивности минимизируется (рис. 8). 


\section{1 ПИТАННЯ ТЕОРІЇ, МЕТОДИ ТА АЛГОРИТМИ ЕФЕКТИВНОГО АВТОМАТИЧНОГО} УПРАВЛІННЯ ОБ’ЄКТАМИ ХІМІКО-ТЕХНОЛОГІЧНОГО ТИПУ

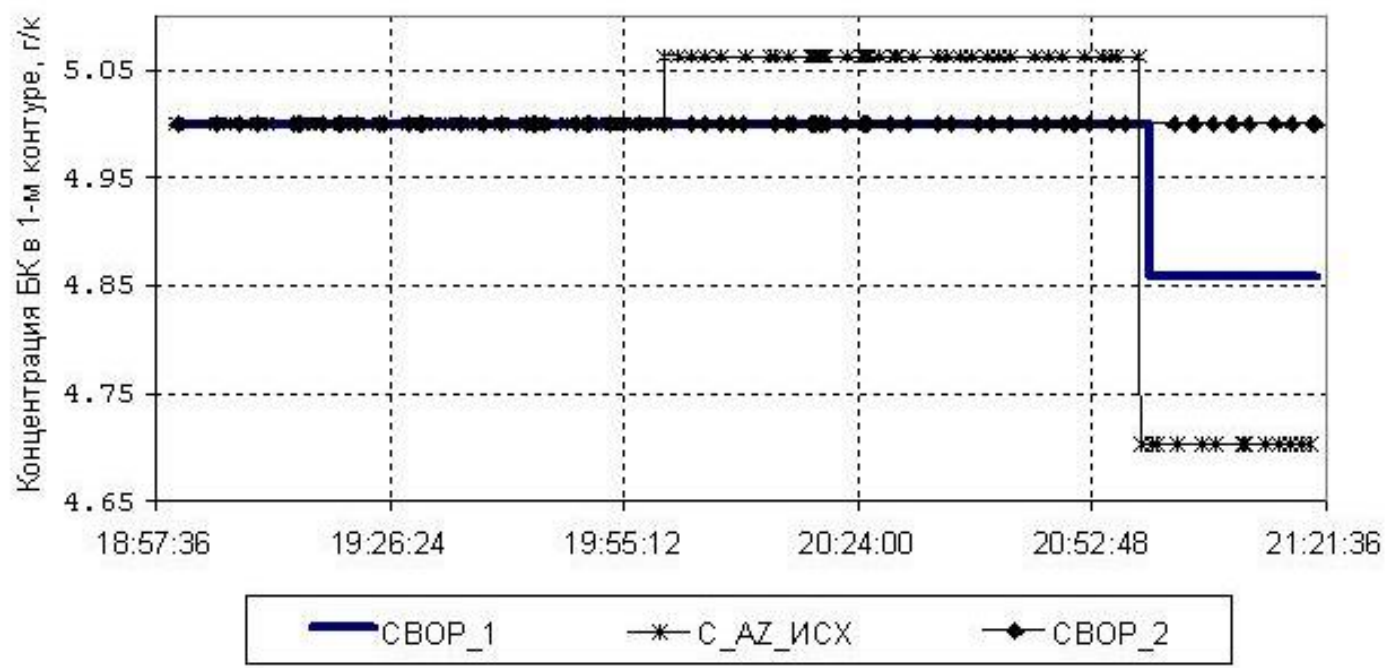

Рис. 7. Изменение концентрации БК в процессе БР

На втором этапе проверки в системе управления была рассмотрена и спрогнозирована ситуация, в которой оператор после срабатывания АРМ не предпринимает никаких действий. Прогноз был выполнен в предположении, что изменение положения ОР СУЗ, уровня мощности и концентрации борной кислоты в контуре не происходит. Изменение реактивности после срабатывания АРМ не происходит, однако ее значение не минимизируется, как это показано на рис. 8 .

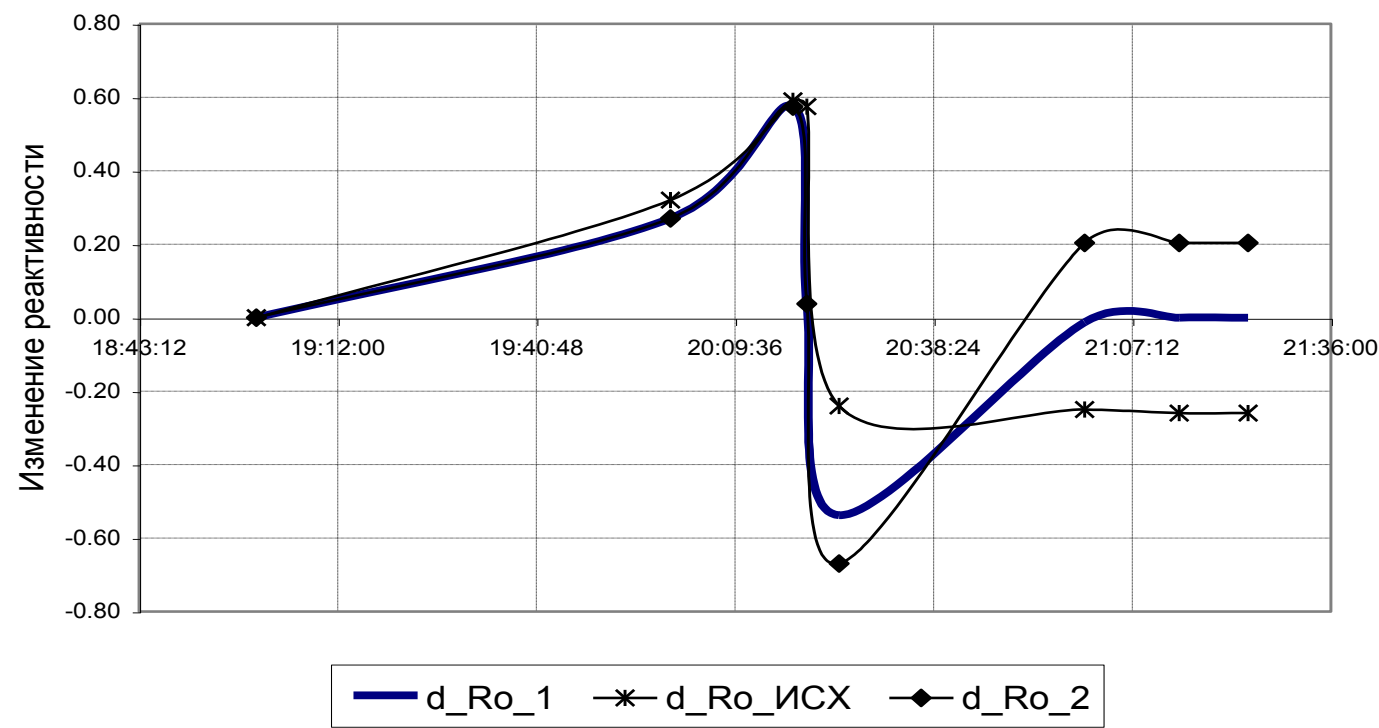

Рис. 8. Изменение реактивности

Выводы. Таким образом, предложен критерий оптимального управления процессом борного регулирования, обеспечивающий минимальное изменение реактивности реактора в процессе управления и поддержание РУ в критическом состоянии. Критерий выражен в виде минимизируемого функционала, проанализированы ограничения, возникающие в процессе управления РУ и определены условия достижения минимума предложенного функционала.

Разработан алгоритм оптимального управления процессом борного регулирования, позволяющий формировать рекомендации оператору с указанием длительности переходного процесса, объема подпитки, значений конечной концентрации борной кислоты в теплоносителе первого контура, а также режима ввода борной кислот либо чистого конденсата. Рекомендация вырабатывается с учетом текущего состояния реакторной установки в реальном времени. 


\section{1 ПИТАННЯ ТЕОРЇ̈, МЕТОДИ ТА АЛГОРИТМИ ЕФЕКТИВНОГО АВТОМАТИЧНОГО УПРАВЛІННЯ ОБ'ЄКТАМИ ХІМІКО-ТЕХНОЛОГІЧНОГО ТИПУ}

Литература

1. Maksimov, M. V. A model of a power unit with WWER-1000 as an object of power control [Text] / M. V. Maksimov, K. V. Beglov, T. A. Tsiselskaya // works of the Odessa Polytechnic University. — Odessa, 2012. Rel. 1(38). - P. 104-106.;

2. Способ стабилизации аксиального распределения нейтронного поля при маневрировании мощностью ВВЭР-1000 / М.В. Максимов, С.Н. Пелых, Т.А. Цисельская, В.Е. Баскаков // Ядерна та радіаційна безпека. - 2011. - Вип. 1(49). - С. 27 - 32.;

3. Демченко, В.А. Автоматизация и моделирование технологических процессов АЭС и ТЭС [Текст] / В.А. Демченко. - О.: Астропринт, 2001. - 308 с.;

4. Цисельская, Т.А. Усовершенствованная АСР энергоблоком реактора / О.Б. Максимова, Т.А. Цисельская // Сборник тезисов докладов 10-й Международной научно-практической конференции по атомной энергетике "Безопасность, эффективность, ресурс". - Севастополь, Оргкомитет МНПК АЭ-10. - С. 33 40.;

5. Maksimov M.V. Model of cladding failure estimation for a cycling nuclear unit / M.V. Maksimov, S.N. Pelykh, O.V. Maslov, V.E. Baskakov // Nuclear Engineering and Design. — 2009. — Vol. 239, № 12. —P. 3021—3026.

6. Метод оценки эксплуатационного ресурса оболочки твэла ВВЭР-1000 в различных режимах нагружения / М.В. Максимов, С.Н. Пелых, О.В. Маслов, В.Е. Баскаков // Атомная энергия. - 2010. - Т. 108, Вып. 5. C. $294-299$;

7. Medvedev R., Sanginova O. Optimization of the boric regulation process at the nuclear power plants of Ukraine // Summaries of 14th International Congrss of Chemical and Process Engineering CHISA-2000/ - Vol. 4. - Praha (Czech Republic). - P. 13.;

8. Сангинова О.В. Разработка технологических алгоритмов борного регулирования для реакторных установок типа ВВЭР-1000 // Вісник Житомирського інженерно-технологічного інституту. — 2001. — № 19. - C. $142-147$.

\section{References}

1. Maksimov, M. V. A model of a power unit with WWER-1000 as an object of power control [Text] / M. V. Maksimov, K. V. Beglov, T. A. Tsiselskaya // works of the Odessa Polytechnic University. — Odessa, 2012. Rel. 1(38). - P. 104-106.;

2. Maksimov M.V. Sposob stabilizatsii aksial'nogo raspredeleniya neytronnogo polya pri manevrirovanii moshchnost'yu WWER-1000 [A method for stabilizing the axial distribution of the neutron field for maneuvering capacity of the WWER-1000] / M.V. Maksimov, S.N. Pelykh, T.A. Tsisel'skaya, V.E. Baskakov // Yaderna ta radiatsiina bezpeka [Nuclear and Radiation Safety]. — 2011. — Iss. 1(49). — pp. 27 - 32.;

3. Demchenko, V.A. Avtomatizatsiya i modelirovanie tekhnologicheskikh protsessov AES i TES [Tekst] [Automation and modeling of NPP and TPP processes [Text]] / V.A. Demchenko. — Odessa, 2001. — 308 p.;

4. Tsisel'skaya, T.A. Usovershenstvovannaya ASR energoblokom reaktora [Advanced RSA of a reactor power unit] / O.B. Maksimova, T.A. Tsisel'skaya // Sbornik tezisov dokladov 10-y Mezhdunarodnoy nauchno-prakticheskoy konferentsii po atomnoy energetike "Bezopasnost', effektivnost', resurs" [Abstracts of the 10th International Scientific and Practical Conference on Nuclear Energy "Safety, efficiency, resource”]. — Sevastopol'. — pp. 33 - 40.;

5. Maksimov M.V. Model of cladding failure estimation for a cycling nuclear unit / M.V. Maksimov, S.N. Pelykh, O.V. Maslov, V.E. Baskakov // Nuclear Engineering and Design. — 2009. — Vol. 239, № 12. —P. 3021—3026.;

6. Pelykh S.N. Metod otsenki ekspluatatsionnogo resursa obolochki tvela WWER-100 v razlichnih rezhimah nagruzheniya [A method of evaluating the performance resource of WWER-1000 fuel cladding in various loading conditions] / M.V. Maksimov, S.N. Pelykh, O.V. Maslov, V.E. Baskakov // Atomnaya energia [Atomic energy]. - 2010. - Iss. 5 - pp. 294 - 299.;

7. Medvedev R., Sanginova O. Optimization of the boric regulation process at the nuclear power plants of Ukraine // Summaries of 14th International Congrss of Chemical and Process Engineering CHISA-2000/ - Vol. 4. - Praha (Czech Republic). - P. 13.;

8. Sanginova O.V. Razrabotka tehnologicheskih algoritmov bornogo regulirovaniya dlia reaktornyh ustanovok tipa WWER-1000 [A development of technological boron control algorithms for reactor units WWER-1000] // Visnyk Zhytomyrs'kogo inzhenerno-tehnologichnogo instytutu [Journal of Zhytomyr Engineering and Technology Institute] — 2001. — №19. — pp. 142 - 147. 